\title{
Inhibition of activated phosphatidylinositol 3-kinase/AKT pathway in malignant pleural mesothelioma leads to G1 cell cycle arrest
}

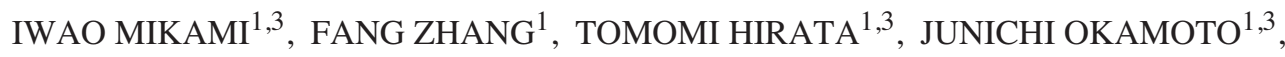 \\ KIYOSHI KOIZUMI ${ }^{3}$, KAZUO SHIMIZU ${ }^{3}$, DAVID JABLONS ${ }^{1,2}$ and BIAO HE HE $^{1,2}$ \\ ${ }^{1}$ Thoracic Oncology Program, Department of Surgery, University of California, San Francisco, CA 94115 \\ ${ }^{2}$ Helen Diller Family Comprehensive Cancer Center, University of California, San Francisco, CA 94143, USA \\ ${ }^{3}$ Department of Surgery, Division of Thoracic Surgery, Nippon Medical School, Tokyo 113-8602, Japan
}

Received April 19, 2010; Accepted July 2, 2010

DOI: 10.3892/or_00001033

\begin{abstract}
Phosphatidylinositol 3-kinase (PI3K)/AKT signaling pathway plays pivotal roles in fundamental cellular functions including cell proliferation and cell survival. Its deregulation has been implicated in many types of human malignancies. We investigated the role of PI3K/AKT signaling pathway in human malignant pleural mesothelioma (MM). Here, we report that aberrant activation of the PI3K/AKT signaling pathway is associated with cell cycle progression in MM cells. Inhibition of the PI3K activity by its small molecule inhibitor LY294002 led to significant G1 cell cycle arrest and suppression of cell proliferation in all MM cell lines that we examined. In addition, we found that the protein level of p27 ${ }^{\text {Kip } 1}$ was up-regulated and the protein level of cyclin D1 was down-regulated following LY294002 treatment in those MM cell lines. However, no noticeable apoptosis induction was observed following $24 \mathrm{~h}$ of LY294002 treatment in those MM cell lines. These results confirm that the PI3K/AKT signaling pathway is aberrantly active and plays a critical role for the cell cycle progression in human MM cells.
\end{abstract}

\section{Introduction}

Malignant pleural mesothelioma (MM) is an asbestos-associated malignancy characterized by rapidly progressive and diffuse growth, late metastases, and very poor prognosis $(1,2)$. Approximately 3000 patients are diagnosed each year in the United States, and the number of incidents is expected

Correspondence to: Dr Biao He, Thoracic Oncology Program, Department of Surgery, Helen Diller Family Comprehensive Cancer Center, University of California, San Francisco, 2340 Sutter Street, Room N222, San Francisco, CA 94115, USA

E-mail: biao.he@ucsfmedctr.org

Key words: PI3K, AKT, G1 arrest, mesothelioma, human to steadily rise and peak around the year $2020(3,4)$. Unfortunately, the disease is of rapid progression and most patients die within 12-15 months of the first symptoms if untreated $(1,5)$. However, the results of standard therapies (surgery, chemotherapy, and radiation) remain dismal. The need to find effective new therapies based on improved understanding of the molecular mechanism of MM remain urgent.

MM shows frequent genetic inactivation of tumor suppressor genes of p16(INK4a)/p14(ARF) and neurofibromatosis type 2 (NF2), and epigenetic inactivation of RASSF1A (6). One case of phosphatase and tensin homologue deleted on chromosome Ten (PTEN) homozygous deletion has been reported in one MM cell line (7). However, no frequent mutations of oncogenes such as K-RAS and PIK3CA have been identified (6). Activation of multiple receptor tyrosine kinases including the epidermal growth factor receptor (EGFR) family and MET, and subsequent deregulations of mitogen-activated protein kinase (MAPK) and phosphatidylinositol-3-kinase (PI3K)-AKT signaling cascades are frequently observed in most MM cells (6). For example, it has been analyzed that tyrosine kinase inhibitors significantly inhibited epidermal growth factordependent cell signaling including phosphorylation of AKT in $\mathrm{MM}$ cell lines $(8,9)$. Furthermore, microarray analysis of MM recently revealed alterations in components of the insulin-like growth factor system, which are associated with the malignant phenotypes (10). PI3K/AKT pathway presents an appealing cancer therapeutic target, as it participates in many fundamental cellular processes including cell survival, cell cycle progression, glucose metabolism, genome stability and angiogenesis (11). Emerging evidence suggests that aberrant PI3K/AKT signaling is one of the most common molecular alterations in human malignancies (11). AKT is the center of a signaling cascade consisting of many components implicated in tumorigenesis, including upstream tyrosine receptor kinases, PI3K, PTEN, NF1 and LKB1, and down-stream tuberous sclerosis complex 2 (TSC2), Forkhead box class $\mathrm{O}$ (FOXO) and eukaryotic initiation factor 4E (eIF4E) (11). Altogether, evidence supports that the PI3K/AKT signaling pathway is dysregulated in MM 
and inhibition of the PI3K activity and/or this signaling pathway may have a therapeutic role in the treatment of MM $(7,12)$.

Disruption of cell cycle checkpoints is frequent in human malignancies, and is often due to deregulation of cyclindependent kinase inhibitors (CKIs). Ink4 family CKIs are lost through deletion, point mutation, and/or promoter methylation. Differently, the Cip/Kip family CKI p27 Kip1 is regulated through protein degradation and cytoplasmic sequestration (13), and its inactivation is fundamental for the tumor development of many types of cancer. Loss of p2 $7^{\text {Kip } 1}$ expression and its cytoplasmic mislocalization are markers for poor prognosis in multiple types of human cancers including malignant mesothelioma (14-17).

PI3K/Akt pathway exerts multiple roles in cell cycle progression through regulating key cell cycle regulators including p27, p21 and cyclin D (18). In this study, we investigated the cell cycle progression after inhibition of the PI3K/AKT signaling in human malignant mesothelioma cells.

\section{Materials and methods}

Cell lines and tissue samples. Human mesothelioma cell lines were obtained from the following sources: H2052, $\mathrm{H} 28$ and $211 \mathrm{H}$ from American Type Culture Collection (Manassas, VA), MS-1 from NIH (Frederick, MD), and REN through a generous gift from Dr Steven Albelda (University of Pennsylvania, PA). All cell lines were cultured in RPMI1640 supplemented with $10 \%$ fetal bovine serum at $37^{\circ} \mathrm{C}$ in a humidified incubator with $5 \% \mathrm{CO}_{2}$. Fresh MM tissue samples obtained directly from surgical resection were immediately snap-frozen in liquid nitrogen. They were preserved at $-170^{\circ} \mathrm{C}$ in a liquid nitrogen before use. Patients consented to tissue specimen collection prospectively, and the study was approved by the University of California, San Francisco Institutional Review Board (CHR\# H871417773-10).

Inhibitor. PI3K inhibitor LY294002 was purchased from Cell Signaling Technology (Beverly, MA). Stock solution was prepared in $100 \%$ DMSO and stored at $-20^{\circ} \mathrm{C}$. The drug was diluted in fresh media before each experiment.

Cell cycle analysis. Cells were plated on 6-well plates, incubated for $24 \mathrm{~h}$, and then treated with DMSO, or LY294002. For flow cytometry, cells were trypsinized and fixed in $70 \%$ ethanol at $-20^{\circ} \mathrm{C}$, washed and stained with $30 \mu \mathrm{g} / \mathrm{ml}$ propidium iodide (Sigma, St. Louis, MO), and then incubated with $10 \mu \mathrm{g} / \mathrm{ml}$ RNase (Roche, Indianapolis, IN) for $1 \mathrm{~h}$ at room temperature. Cells were evaluated on a FACScan machine (Becton-Dickinson, Franklin Lake, NJ) and the data analyzed with the ModFit LT 3.1 Mac software for modeling cell cycle distribution. Experiments were performed in triplicate, and data was expressed as mean $\pm \mathrm{SD}$.

Cell proliferation assay. Cell proliferation assays were performed using the CellTiter 96 AQueous One Solution Cell Proliferation Assay (Promega, Madison, WI) according to the manufacturer's protocol. Cells were plated at 1000 cells/well in $100 \mu 1$ of media in 96-well plates. After $24 \mathrm{~h}$, the cells were treated with LY294002 and incubated for $48 \mathrm{~h}$. Methanethoiosulfonate/phenazine methosulfate solution (20 $\mu \mathrm{l} /$ well $)$ was added and incubated for $2 \mathrm{~h}$ at $37^{\circ} \mathrm{C}$ in a humid incubator with $5 \% \mathrm{CO}_{2}$. Absorbance was read at $490 \mathrm{~nm}$ using a microplate reader. Cell viability was calculated according to the following formula. Cell viability = OD490 (DMSO or LY294002 treated cells)/OD490 (non-treated cells).

Western blotting. Whole cell lysates of MM cell lines and primary tissue cultures were obtained using CytoBuster Protein Extraction Reagent (Novagen, Madison, WI). Protein samples were separated on 4-15\% gradient SDS-polyacrylamide gels and transferred to Immobilion-P (Millipore, Bedford, MA) membranes. Antigen-antibody complexes were detected by the ECL blotting analysis system (Amersham Pharmacia Biotech, Piscataway, NJ). Primary antibodies used include: phosphorylated AKT (Cell Signaling Technology), AKT and Cyclin D1 (Santa Cruz Biotechnology, Santa Cruz, CA), p27 and $\beta$-actin (Sigma).

Statistical analysis. The data are presented as mean values \pm SD. Statistical comparisons were made with a two-sided Student's t-test. A p-value of $<0.05$ was considered to be statistically significant.

\section{Results}

Confirmation of the PI3K/AKT activation in MM cell lines and primary tissue samples. To investigate whether the $\mathrm{PI} 3 \mathrm{~K} / \mathrm{AKT}$ signaling pathway is activated in MM, we first examined the level of the activated serine 476-phosphorylated AKT (p-AKT) protein in $5 \mathrm{MM}$ cell lines (MS-1, REN, 211H, H28, and H2052). These cell lines were grown in serum free medium for $24 \mathrm{~h}$ and were treated with $10 \%$ fetal bovine serum (FBS) for $10 \mathrm{~min}$. The expression level of phosphorylated AKT protein was compared before and after FBS treatment. We found that level of phosphorylated AKT protein was up-regulated following serum treatment compared to serum free treated cells in all MM cell lines (Fig. 1A). Next, we performed Western blot analysis using fresh primary MM tissue samples obtained with consent from patients undergoing resection, to examine the existence of the phosphorylated AKT. Among the 20 fresh MM tissue samples that we examined, 95\% (19/20) showed expression of phosphorylated AKT protein (Fig. 1B). These results confirm that the $\mathrm{PI} 3 \mathrm{~K} / \mathrm{AKT}$ signaling pathway is active in human MM.

Inhibition of PI3K led to G1 cell cycle arrest in MM cells. Although the PI3K/AKT signaling pathway has been observed to play an important role in human cancer cells (19-21), its role in cell cycle controlling in human mesothelioma is still not well understood. We examined the role of PI3K/AKT signaling pathway in cell cycle controlling in MM using the PI3K inhibitor, LY294002. To determine the optimal concentration of LY294002 to inhibit the phosphorylation of AKT in MM cell lines, $211 \mathrm{H}$ cell line was treated with varying concentrations of LY294002 for $1 \mathrm{~h}$ and its effect on phosphorylated AKT protein level was 


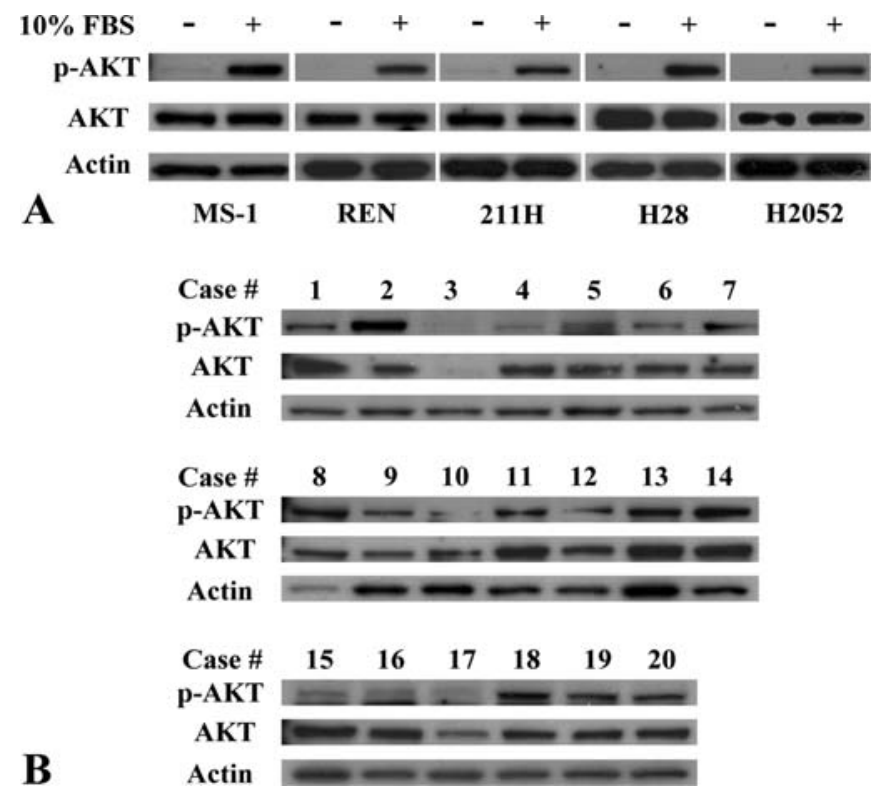

Figure 1. Activation of the PI3K/AKT signaling pathway in human MM. (A) Western blot analysis of phosphorylated AKT in 10\% serum treated MM cell lines compared with serum free treated ones. (B) Western blot analysis of the phosphorylated AKT in fresh primary MM tissue samples.
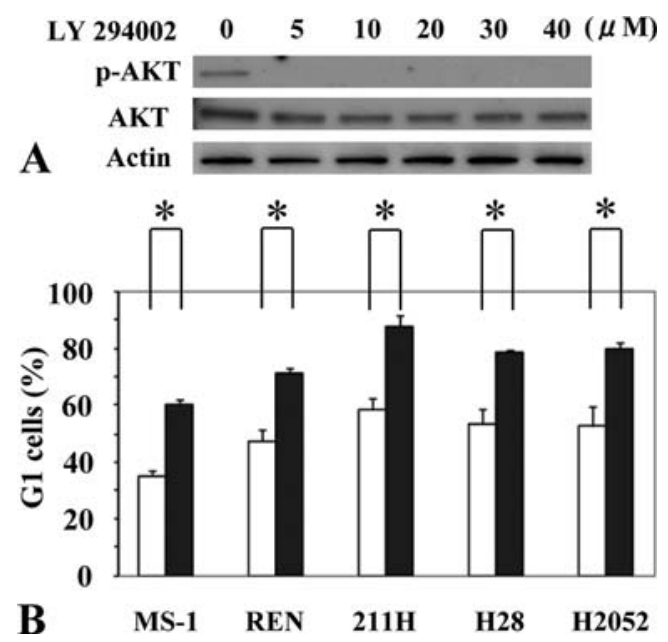

Figure 2. PI3K inhibitor leads to G1 cell cycle arrest in MM cell lines. (A) Western blot of phosphorylated AKT in $211 \mathrm{H}$ cells after treated with 0-40 $\mu \mathrm{M}$ LY294002 for $1 \mathrm{~h}$. (B) Cell cycle analysis in LY294002 and DMSO treated cells. Cells were plated in 6-well plates, incubated for $24 \mathrm{~h}$, and treated with DMSO or $20 \mu \mathrm{M}$ LY294002 in the presence of $10 \%$ FBS. Experiments were performed in triplicate. Data are means \pm SD (white bars, DMSO; black bars, LY294002). Asterisks represent statistical significance $(\mathrm{p}<0.05)$.

assayed. Phosphorylated AKT protein was dramatically down-regulated at varying concentrations between 5-40 $\mu \mathrm{M}$ in LY294002 treated cells (Fig. 2A). The same result was also observed in other $4 \mathrm{MM}$ cell lines (data not shown). Next, we treated all five MM cell lines with $20 \mu \mathrm{M} \mathrm{LY} 294002$ for $24 \mathrm{~h}$ and performed cell cycle analysis by using FACS. Significant G1 cell cycle arrest was observed in LY294002 treated cells compared to DMSO treated cells in all MM cell lines (Fig. 2B). Consistently, MTT assays after treatment with a

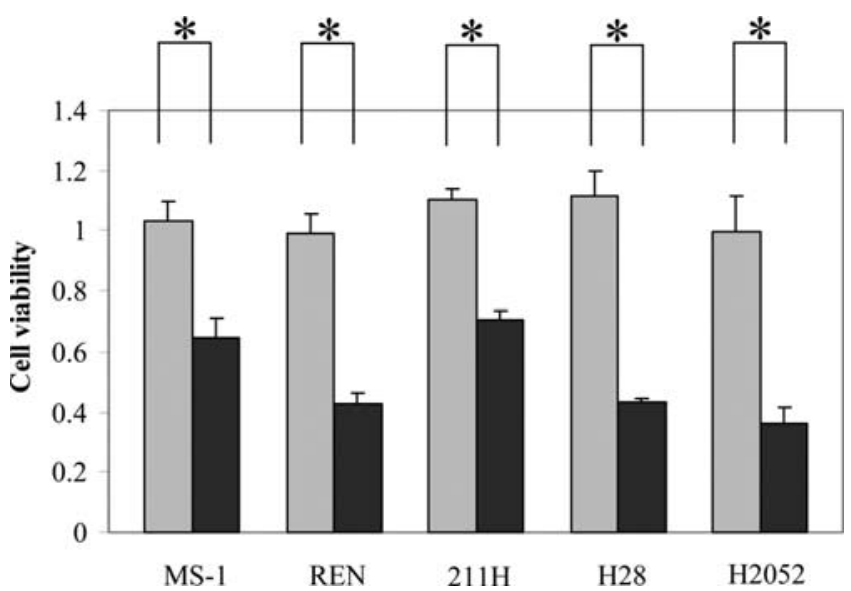

Figure 3. Cell proliferation assay in MM cells after treated with PI3K inhibitor LY294002 $(20 \mu \mathrm{M})$ in the presence of $10 \%$ FBS and incubated for $72 \mathrm{~h}$. Experiments were performed in triplicate. Data are the means \pm SD (gray bars, DMSO treated cells; black bars, LY294002 treated cells). Asterisks represent statistical significance $(\mathrm{p}<0.05)$.

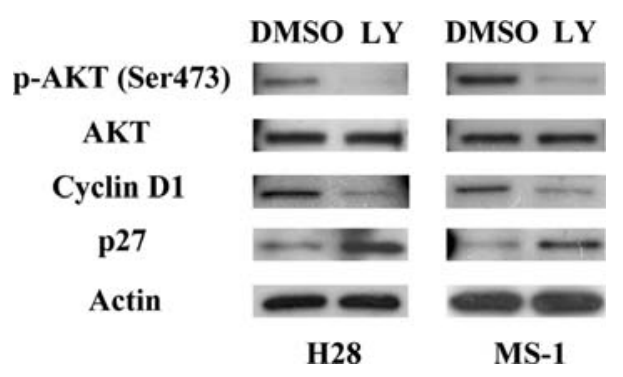

Figure 4. Western blot analysis of cell cycle proteins in MM cell lines. Whole-cell lysate was used after cells were treated with DMSO or LY294002 $(20 \mu \mathrm{M})$ in the presence of $10 \%$ FBS for $24 \mathrm{~h}$. Actin served as a loading control.

concentration of $20 \mu \mathrm{M}$ LY294002 for $72 \mathrm{~h}$ showed significant inhibition of cell proliferation in LY294002 treated cells compared to DMSO treated cells in all MM cell lines (Fig. 3). Taken together, our results suggest that cell proliferation suppression by inhibition of the PI3K/AKT signaling pathway is strongly associated with G1 cell cycle arrest in human MM.

PI3K/AKT signaling drives cell cycle progression through regulating the stability and activity of the cyclin-dependentkinase $(\mathrm{CDK})$ inhibitor such as $\mathrm{p} 27^{\mathrm{Kip} 1}$ and cyclin D1 $(18,20)$. To examine the effect of PI3K inhibition of key regulators of cell cycle progression, we treated the MM cells with $20 \mu \mathrm{M}$ LY294002 for $24 \mathrm{~h}$, which significantly inhibited AKT phosphorylation in the MM cells, and steady state level of p27 and cyclin D1 level was evaluated by Western blot analysis (Fig. 4, results in the other three cell lines were the same as those shown in H28 and MS-1). Remarkably, p27 Kip1 protein level was significantly up-regulated after LY294002 treatment in MM cell lines. Furthermore, protein level of cyclin D1 was significantly down-regulated in LY294002 treated cell lines. Therefore, our data suggest that in MM the PI3K/AKT signaling pathway may regulate cell cycle through key regulators such as cyclin D1 and p27 Kip1. 


\section{Discussion}

The PI3K/AKT signaling pathway is frequently deregulated in many types of human malignancies and plays a major role in both tumorigenesis and response to chemotherapy (22). A better understanding of the status of the PI3K/AKT signaling pathway in malignant mesothelioma may improve therapeutic intervention and prognosis in refractory MM. In this study, we confirmed that the PI3K/AKT signaling pathway was hyperactive in human $\mathrm{MM}$, and demonstrated that inhibition of PI3K signaling induced significant G1 cell cycle arrest and up-regulated CDK inhibitors p27 and down-regulated cyclin D1. The data strongly support that the PI3K/AKT signaling pathway regulates cell cycle progression and cell proliferation in malignant mesothelioma.

Our data indicating elevated activity of AKT as shown by the phosphorylated (Ser 476) form of AKT in primary mesothelioma samples, are consistent with a previous report on PI3K/AKT signaling hyperactivity in human and mouse mesothelioma (7). More importantly, our data that inhibition of PI3K activity with its specific inhibitor LY294002 for $24 \mathrm{~h}$ significantly induced cell cycle arrest at G1/S boundary strongly support that PI3K/AKT pathway is essential for regulating cell cycle progression in human mesothelioma. We did not observe significant apoptosis induction in the mesothelioma cell lines that we examined after treating with LY294002 for up to $24 \mathrm{~h}$. However, prolonged treatment with LY294002 for up to $72 \mathrm{~h}$ resulted in significantly lower viability in these cells, consistent with the previous report that prolonged treatment (up to $48 \mathrm{~h}$ ) of LY294002 induced apoptotic cell death in mesothelioma cells (7). Taken together, our results suggest that prolonged inhibition of the PI3K/AKT pathway may shut down cell survival network, while short-term inhibition of PI3K/AKT signaling suppresses cell cycle progression.

Burgeoning literature has linked the PI3K/AKT path-way to regulation of the main cell cycle machinery (18-20). Activated AKT triggers a network that positively drives G1/S cell cycle progression through inactivation of GSK-3ß, leading to increased cyclin D1 (23). Consistently, we observed decreased cyclin D1 protein following inhibition of PI3K activity in MM cells. Activated PI3K/AKT pathway can lead to reduction of $\mathrm{p} 27^{\mathrm{Kip} 1}$ protein level via increased $\mathrm{p} 27$ proteolysis through its ubiquitin ligase Skp2 (18,24-26). Consistently, we showed remarkable increase of p27 protein level following inhibition of the PI3K activity in MM cells. As cyclin D1 is a proto-oncogene that promotes Cdk4 and Cdk6 activity in order to drive cell cycle progression through G1/S, and p27 suppresses cyclin D-Ckd4 and cyclin E-Cdk2 activity, the reduction of cyclin D1 and increase of p27 correlate with the G1 arrest following PI3K inhibition in MM cells. Taken together, these results demonstrate that PI3K/AKT signaling pathway plays a critical role in driving cell cycle progression via modulating key regulators of cell cycle machinery.

In summary, we confirmed that the PI3K/AKT signaling pathway is frequently activated in human MM cell lines and tissue samples. We also showed that the specific PI3K inhibitor, LY294002, led to G1 cell cycle arrest and suppression of cell growth in human MM cell lines. Our findings shed light on an unappreciated role of the PI3K/AKT pathway in regulating cell cycle progression in human mesothelioma, and support this pathway as a therapeutic target for this highly aggressive and inexorably cancer.

\section{Acknowledgements}

Grant Support: NIH/NCI Grant R01CA125030, Joan's Legacy: Uniting Against Lung Cancer Research Grant, and the Eileen D. Ludwig Endowed for Thoracic Oncology Research (to B.H.); the Bonnie J. Addario Lung Cancer Foundation, the Kazan, McClain, Abrams, Fernandez, Lyons, Greenwood, Harley \& Oberman Foundation, and the Barbara Isackson Lung Cancer Research Fund (D.J.).

\section{References}

1. Belli C, Fennell D, Giovannini M, Gaudino G and Mutti L: Malignant pleural mesothelioma: current treatments and emerging drugs. Expert Opin Emerg Drugs 14: 423-437, 2009.

2. Yang CT, You L, Uematsu K, Yeh CC, McCormick F and Jablons DM: p14(ARF) modulates the cytolytic effect of ONYX-015 in mesothelioma cells with wild-type p53. Cancer Res 61: 5959-5963, 2001.

3. Price B: Analysis of current trends in United States mesothelioma incidence. Am J Epidemiol 145: 211-218, 1997.

4. Peto J, Decarli A, La Vecchia C, Levi F and Negri E: The European mesothelioma epidemic. Br J Cancer 79: 666-672, 1999.

5. Tomasetti M, Amati M, Santarelli L, Alleva R and Neuzil J: Malignant mesothelioma: biology, diagnosis and therapeutic approaches. Curr Mol Pharmacol 2: 190-206, 2009.

6. Sekido Y: Genomic abnormalities and signal transduction dysregulation in malignant mesothelioma cells. Cancer Sci 101: $1-6,2010$.

7. Altomare DA, You H, Xiao GH, Ramos-Nino ME, Skele KL, De Rienzo A, Jhanwar SC, Mossman BT, Kane AB and Testa JR: Human and mouse mesotheliomas exhibit elevated AKT/PKB activity, which can be targeted pharmacologically to inhibit tumor cell growth. Oncogene 24: 6080-6089, 2005.

8. Janne PA, Taffaro ML, Salgia R and Johnson BE: Inhibition of epidermal growth factor receptor signaling in malignant pleural mesothelioma. Cancer Res 62: 5242-5247, 2002.

9. Nutt JE, O'Toole K, Gonzalez D and Lunec J: Growth inhibition by tyrosine kinase inhibitors in mesothelioma cell lines. Eur J Cancer 45: 1684-1691, 2009.

10. Hoang CD, Zhang X, Scott PD, Guillaume TJ, Maddaus MA, Yee D and Kratzke RA: Selective activation of insulin receptor substrate-1 and -2 in pleural mesothelioma cells: association with distinct malignant phenotypes. Cancer Res 64: 7479-7485, 2004.

11. Bellacosa A, Kumar CC, Di Cristofano A and Testa JR: Activation of AKT kinases in cancer: implications for therapeutic targeting. Adv Cancer Res 94: 29-86, 2005.

12. Cole GW Jr, Alleva AM, Zuo JT, Sehgal SS, Yeow WS, Schrump DS and Nguyen DM: Suppression of pro-metastasis phenotypes expression in malignant pleural mesothelioma by the PI3K inhibitor LY294002 or the MEK inhibitor UO126. Anticancer Res 26: 809-821, 2006.

13. Lee J and Kim SS: The function of p27 KIP1 during tumor development. Exp Mol Med 41: 765-771, 2009.

14. Beer TW, Sheperd P and Pullinger NC: p27 immunostaining is related to prognosis in malignant mesothelioma. Histopathology 38: 535-541, 2001

15. Bongiovanni M, Cassoni P, De Giuli P, Viberti L, Cappia S, Ivaldi C, Chiusa L and Bussolati G: p27(kip1) immunoreactivity correlates with long-term survival in pleural malignant mesothelioma. Cancer 92: 1245-1250, 2001.

16. Viglietto G, Motti ML and Fusco A: Understanding p27(kip1) deregulation in cancer: down-regulation or mislocalization. Cell Cycle 1: 394-400, 2002.

17. Slingerland J: Transforming growth factor-beta and breast cancer: introduction. Breast Cancer Res 2: 91, 2000. 
18. Liang $\mathbf{J}$ and Slingerland JM: Multiple roles of the PI3K/PKB (Akt) pathway in cell cycle progression. Cell Cycle 2: 339-345, 2003.

19. Cantley LC: The phosphoinositide 3-kinase pathway. Science 296: 1655-1657, 2002

20. Nicholson KM and Anderson NG: The protein kinase B/Akt signalling pathway in human malignancy. Cell Signal 14: 381-395, 2002 .

21. Franke TF, Hornik CP, Segev L, Shostak GA and Sugimoto C: PI3K/Akt and apoptosis: size matters. Oncogene 22: 8983-8998, 2003.

22. Fresno Vara JA, Casado E, de Castro J, Cejas P, Belda-Iniesta C and Gonzalez-Baron M: PI3K/Akt signalling pathway and cancer. Cancer Treat Rev 30: 193-204, 2004.
23. Diehl JA, Cheng M, Roussel MF and Sherr CJ: Glycogen synthase kinase-3beta regulates cyclin D1 proteolysis and subcellular localization. Genes Dev 12: 3499-3511, 1998.

24. Montagnoli A, Fiore F, Eytan E, Carrano AC, Draetta GF, Hershko A and Pagano M: Ubiquitination of p27kip 1 is regulated by $\mathrm{Cdk}$-dependent phosphorylation and trimeric complex formation. Genes Dev 13: 1181-1189, 1999.

25. Carrano AC, Eytan E, Hershko A and Pagano M: SKP2 is required for ubiquitin-mediated degradation of the CDK inhibitor p27Kip1. Nat Cell Biol 1: 193-199, 1999.

26. Pagano M, Tam SW, Theodoras AM, Beer-Romero P, Del Sal G, Chau V, Yew PR, Draetta GF and Rolfe M: Role of the ubiquitinproteasome pathway in regulating abundance of the cyclindependent kinase inhibitor p27Kip1. Science 269: 682-685, 1995. 